\title{
Facteurs de variation de la production de méthane au cours de la digestion des aliments composés chez les ruminants
}

\author{
$S$ Giger-Reverdin ${ }^{1}, M$ Vermorel $^{2}$, D Sauvant ${ }^{1}$ \\ 1 INRA, station de nutrition et alimentation, \\ Institut national agronomique Paris-Grignon, 16, rue Claude-Bernard, 75231 Paris Cedex 05; \\ 2 INRA, laboratoire d'étude du métabolisme énergétique, \\ centre de recherche de Clermont-Ferrand-Theix, 63122 Ceyrat, France
}

\begin{abstract}
Summary - Factors of variation of methane energy losses in ruminants given compound feeds. Methane energy losses were measured in wethers for 41 compound feeds. Results showed that the ether extract content of concentrates had a significant negative influence on methanogenesis when associated with one of the other prediction criteria (energy digestibility, crude fibre or lignin content, or enzymatic degradability).
\end{abstract}

Dans le système français des unités fourragères, les pertes d'énergie sous forme de méthane ( $E G$ ) sont estimées de façon cumulée avec celles de l'urine, par une relation établie à partir des résultats obtenus avec 346 rations, qui ne prend en compte que les teneurs en cellulose: brute $(C B)$ et en matières azotées (MAT) (Vermorel et al, 1987). Par rapport à ces rations, les aliments composés contiennent moins de constituants pariétaux, mais plus d'amidon, de matières azotées et de matières grasses (MG). Or, les $M G$ exercent un effet inhibiteur sur la méthanogénèse (Czerkawski et al, 1966). Le présent travail a donc été entrepris pour évaluer et hiérarchiser les facteurs de variation des pertes de EG dans le cas des aliments composés $(A C)$.

Matériel et méthodes - Quarante ef un aliments composés ont fait l'objet de mesures de digestibilité et de production de méthane. Pour cela, des rations constituées, sur la base de la matière sèche $(M S)$, de $60 \%$ d' $A C$ et de $40 \%$ de foin (17 $A C$ à I'INRA de Theix) ou de 25,50 ou $75 \%$ d' $A C$ associés à du foin (24 $A C$ au Rowett Research Institute; Wainman et al, 1981) ont été distribuées en quantité limitée à des moutons à l'entretien maintenus dans des chambres respiratoires. La production EG a été calculée par différence (INRA de Theix), connaissant celle du foin seul, ou par régression (RRI). Les analyses chimiques ou enzymatiques des échantillons ont toutes été faites dans un même laboratoire.

Résultats et discussion - Les caractéristiques analytiques et la digestibilité de l'énergie ( $\mathrm{d} E$ en \%) des aliments figurent au tableau I. EG (en \% de l'énergie digestible, $E D$ ) est en moyenne de $10,7 \%$ avec un écart type de $1,1 \%$. Elle varie entre 8,18 et 13,14 et diminue quand $\mathrm{d} E$ augmente.

$$
\begin{gathered}
E G(\% / E D)=16,3-0,0717 \mathrm{~d} E \\
(0,0277) \\
(r=0,38 ; n=41 ; E T R=1,00)
\end{gathered}
$$

\begin{tabular}{|c|c|c|c|c|c|}
\hline & & Moyenne & Écart type & Minimum & Maximum \\
\hline $\left.\begin{array}{l}C B \\
M A T \\
M G \\
A D L \\
\mathrm{~d} E Z I \\
\mathrm{~d} E Z T \\
\mathrm{~d} E\end{array}\right\}$ & $\left.\begin{array}{l}(\% / M O \\
\% / M O \\
\% / M O \\
\% / M O \\
\% \\
\% \\
\%\end{array}\right)$ & $\begin{array}{r}10,8 \\
22,1 \\
3,7 \\
2,9 \\
77,7 \\
83,5 \\
78,3\end{array}$ & $\begin{array}{l}4,2 \\
6,9 \\
1,7 \\
1,6 \\
6,7 \\
7,1 \\
5,7\end{array}$ & $\begin{array}{r}3,8 \\
14,5 \\
0,8 \\
0,5 \\
60,6 \\
64,7 \\
63,9\end{array}$ & $\begin{array}{r}19,0 \\
51,8 \\
8,0 \\
7,7 \\
90,6 \\
95,8 \\
89,7\end{array}$ \\
\hline
\end{tabular}

Tableau I. Principales caractéristiques des 41 aliments étudiés. 
La valeur du coefficient de régression $(0,072)$ est proche de celle $(0,077)$ obtenue par Blaxter et Clapperton (1965) en compilant les données de 615 régimes ayant une $d E$ très variable (de 50 à $90 \%$ ) et un rapport EG/ED variant de 10,3 à $13,4 \%$, mais une teneur faible en $M G$. La prise en compte de cette teneur en $M G$ (en $\% / M O)$ des échantillons permet d'améliorer significativement l'explication des variations du rapport $E G / E D$ à partir de $d E$ :

$$
\begin{gathered}
E G(\% / E D)=19,5-0,274 M G-0,099 \mathrm{~d} E[2] \\
(0,040) \quad(0,027) \\
(r=0,56 ; n=41 ; E T R=0,90)
\end{gathered}
$$

Le coefficient de régression de la MG $(0,274)$ est très voisin de celui qui peut être calculé à partir des résultats de 4 essais de supplémentation en $M G$ de rations de vaches laitières, publiés par Van der Honing et al (1983) : 0,247. Une partie de l'effet de la MG sur le rapport $E G / E D$ est liée à son influence positive sur la teneur en $E D$ des aliments car son effet sur EG/MOD est moins net d'un point de vue statistique.

La teneur en $C B(\% M O)$ influe aussi significativement sur le rapport $E G / E D$ :

$$
\begin{gathered}
E G(\% / E D)=9,77+0,0873 C B \\
(0,0377) \\
(r=0,35 ; n=41 ; E T R=1,01)
\end{gathered}
$$

La valeur du coefficient de régression est à rapprocher de celui $(0,099$ par point de $C B \%$ de la matière sèche (MS)) obtenu par Vermorel et al (1987). La prise en compte des teneurs en $M G$ ou MAT n'améliore pas cette équation.

La teneur en lignine $(A D L$ en \%/MO) explique mieux les variations de $E G / E D$ que la $C B$. En outre, son association avec $M G$ conduit à une précision équivalente à celle obtenue avec $d E$ associée à $M G$ :

$$
\begin{array}{r}
E G(\% / E D)=10,70-0,270 M G+0,346 A D L \\
(0,091) \quad(0,099) \quad[4] \\
(r=0,54 ; n=41 ; E T R=0,92)
\end{array}
$$

Les autres constituants de la paroi cellulaire comme le NDF ou l'ADF n'apportent pas de gain de précision.

Les dégradabilités enzymatiques (en $\%)$, effectuées selon les méthodes de Theix (dEZT), ou de I'INAPG (dEZI), conduisent à une précision assez comparable :

$$
\begin{aligned}
& E G(\% / E D)=19,38-0,307 M G-0,0902 \mathrm{~d} E Z T \\
& (0,087) \quad(0,0207) \quad[5] \\
& (r=0,62 ; n=41 ; E T R=0,86) \\
& E G(\% / E D)=18,81-0,292 \text { MG-0,0904dEZI } \\
& (0,088) \quad(0,0228) \quad[6] \\
& (r=0,59 ; n=41 ; E T R=0,88)
\end{aligned}
$$

Ces 4 dernières équations permettent d'estimer les pertes d'énergie sous forme de méthane à partir de mesures effectuées au laboratoire. Ainsi, pour une teneur en $d E$ de $83 \%$, la prise en compte de la teneur en MG d'un échantillon influe peu sur le rapport $E G$ l $E D(+0,7 \%)$, ou sur la valeur UFL $(-0,01 \mathrm{UFL} / \mathrm{kg} M O)$ si la teneur en $M G$ est de $0,8 \% / M O$, mais les variations sont plus importantes et en sens inverse si la teneur en $M G$ est de $8 \%$ : $-1,2 \%$ pour $E G / E D$ et $+0,03$ UFL/kg $M O$. La hiérarchie observée entre ces méthodes est comparable à celle de la prédiction de $\mathrm{d} E$.

En conclusion, ces résultats montrent donc que les variations des teneurs en $M G$ des aliments composés ont une influence sensible sur la méthanogenèse, alors qu'elles n'en n'avaient pas sur la digestibilité de la matière organique.

Blaxter KL, Clapperton JL (1965) Br J Nutr 19, 511-522

Czerkawski JW, Blaxter KL, Wainman FW (1966) Br J Nutr 20, 349-362

Van der Honing $Y$, Tamminga $S$, Wieman $B J$, Steg A, Van Donselaar B, Van Gils LGM (1983) Neth J Agric Sci 31, 27-36

Vermorel $M$, Coulon JB, Journet $M$ (1987) Bull Tech CRZV Theix 70, 9-18

Wainman FW, Dewey PJS, Boyne AW (1981) Rowett Research Institute, Report $n^{\circ} 3$, $49 p$ 